(1)

CrossMark

\title{
Aminopeptidase antibodies in mesothelioma: new wine deserves new sacks
}

\author{
Jan P. van Meerbeeck ${ }^{1}$, Gerrit Jansen ${ }^{2}$, Elisa Giovannetti ${ }^{3}$ and \\ Godefridus J. Peters ${ }^{3}$
}

Affiliations: ${ }^{1}$ Dept of Pulmonology and Thoracic Oncology, Antwerp University Hospital and Antwerp University, Edegem, Belgium. ${ }^{2}$ Amsterdam Rheumatology and Immunology Center, VU University Medical Center, Amsterdam, The Netherlands. ${ }^{3}$ Dept of Medical Oncology, VU University Medical Center, Amsterdam, The Netherlands.

Correspondence: Jan P. van Meerbeeck, Dept of Pulmonology and Thoracic Oncology, Antwerp University Hospital and Antwerp University, Edegem, Belgium. E-mail: jan.van.meerbeeck@uza.be

@ERSpublications

Aminopeptidase antibodies are a new class of agents in mesothelioma; their development will require strategic clinical trials. Exploring activity and efficacy according to old paradigms may lead to their disruptive characteristics being disregarded. http://ow.ly/3XLU30jUpqf

Cite this article as: van Meerbeeck JP, Jansen G, Giovannetti E, et al. Aminopeptidase antibodies in mesothelioma: new wine deserves new sacks. Eur Respir J 2018; 51: 1800817 [https://doi.org/10.1183/ 13993003.00817-2018].

In this issue of the European Respiratory Journal, OTSUк et al. [1] postulate that antibody targeting of aminopeptidase $\mathrm{N}$ (APN)/CD13 by MT95-4 might be a potential therapeutic approach for malignant pleural mesothelioma (MPM). Aminopeptidases have several functions, but their main role is breakdown of small peptides [2]. However, aminopeptidases may also play a role in angiogenesis. Angiogenesis signalling is an important hallmark of solid cancer pathophysiology in general and MPM in particular [3]. Several mechanisms are involved in this process; one of the most important factors is the shedding by the tumour of the ligand vascular endothelial growth factor (VEGF), which then binds to the homologous receptor (VEGFR) on blood vessels. This signal leads to neo-angiogenesis: the sprouting of fragile, distorted and aberrant feeding vessels to the tumour. MPM has amongst the highest expression of VEGF and an increased vascularisation is a poor prognostic factor [4].

APN is a $\mathrm{Zn}^{2+}$-dependent membrane-associated ectopeptidase that preferentially cleaves small peptides (2-6 amino acids) with an N-terminal neutral amino acid [5]. CD13 belongs to a family of other aminopeptidases of which puromycin-sensitive aminopeptidase, leukotriene $\mathrm{A}_{4}$ hydrolase, endoplasmic reticulum aminopeptidase $1 / 2$ and leucine aminopeptidase are the main representatives [6]. These aminopeptidases have different cellular localisations (membrane, cytoplasm and subcellular organelles), where they function in protein/peptide degradation and regulate many downstream processes thereof. For CD13 these processes extend to immune functions (antigen presentation), angiogenesis and metastasis [7]. In the plasma membrane, CD13 resides in cholesterol-rich microdomains called lipid rafts in which multiple signalling-associated proteins compartmentalise, thus proposing a role for CD13 in signalling processes [8]. APN is also selectively expressed as a receptor on endothelial cells of the angiogenic vasculature, but not in blood vessels of normal tissues [9]. Collectively, it can be concluded that either increased $\operatorname{VEGF}(\mathrm{R})$ or APN expression are related to a more malignant phenotype. 
In view of their broad function, aminopeptidases, including CD13, have been recognised as chemotherapeutic targets. Currently, inhibitors of aminopeptidases comprise of direct inhibitors, of which bestatin (Ubenimex) is the best known clinically active drug [8], and prodrug inhibitors of aminopeptidases, of which tosedostat is the most promising [10]. Mechanistically, these aminopeptidase inhibitors introduce an intracellular amino acid depletion and inhibition of mammalian target of rapamycin (mTOR) activity, thereby impairing cell proliferation [11]. mTOR is a downstream target of AKT (protein kinase B). AKT acts as a survival/escape pathway. Treatment with drugs, such as cisplatin and pemetrexed, induces phosphorylation and activation of the AKT pathway, which will reduce their efficacy [12]. Since inhibition of AKT can enhance the efficacy of these drugs, conceivably, inhibition of an aminopeptidase can enhance the sensitivity to cisplatin. Whether these mechanistic effects are introduced for the CD13 antibody MT95-4 remains to be determined.

How can a treatment directed against an aminopeptidase be integrated with the current treatment of MPM? The most widely used standard treatment is a combination of the antifolate drug pemetrexed and a platinum analogue either cisplatin or carboplatin. GiovANNETTI et al. [13] recently reported that the efficacy of pemetrexed is dependent on the activity of both its target thymidylate synthase and its influx transporter (PCFT). In fact, a high expression of PCFT and a low expression of thymidylate synthase were associated with a better survival. There is no evidence indicating that CD13 would play a direct role in the expression and functioning of these targets. However, since inhibition of the upstream AKT enhances the effect of pemetrexed and cisplatin, an aminopeptidase inhibitor might be able to enhance the efficacy of both cisplatin (and carboplatin) and pemetrexed. A potent synergistic interaction was found between carboplatin-pemetrexed and the EGFR/VEGFR-2/RET inhibitor vandetanib against MPM cell lines [14]. Several factors contributed to this synergistic interaction including apoptosis induction and modulation of phosphorylation and expression of critical gene products involved in drug activity/resistance (i.e. downregulation of phospho-Akt and of ERCC1 and XPD expression). Further studies should clarify whether CD13 targeting would be of added value to this combination, e.g. by inhibition of both the pemetrexed/cisplatin-induced stimulation of the AKT pathway, or would CD13's role be more related to inhibition of angiogenesis? Of note, since MT95-4 does not recognise murine APN/CD13, the anti-tumour and anti-angiogenic effects of MT95-4 described by OTsUKI et al. [1] were achieved by inhibiting the activity of APN/CD13 expressed only in tumour cells. However, when used for patients with MPM, MT95-4 may exert stronger anti-tumour effects through inhibition of APN/CD13 activity in both tumour and stromal cells.

Several trials have investigated the efficacy of intravenous anti-VEGF(R) agents in mesothelioma. In the large, phase 3 MAPS trial bevacizumab, a monoclonal antibody against circulating VEGF, was added to first line cisplatin-pemetrexed backbone in 448 treatment naïve patients [15]. Both progression-free survival (PFS) and overall survival were significantly increased in that trial, but not in a similar one adding bevacizumab to a cisplatin-gemcitabine combination [16]. The reason for this is unclear and possibly drug-class related. Similar discrepancies in outcome were observed in advanced nonsmall cell lung cancer where a combination of bevacizumab with paclitaxel and carboplatin showed a significant outcome benefit, whereas a combination with gemcitabine and cisplatin did not $[17,18]$. Moreover, a phase 2 study of pemetrexed and carboplatin plus bevacizumab as first-line therapy in MPM did not show the predicted improvement in median PFS in comparison with a standard pemetrexed/platinum combination [19]. However, due to the observed relatively small increase in overall survival, the addition of bevacizumab to chemotherapy has not become standard of care in most parts of the world, and is recommended as optional in the National Cancer Center Network guidelines [20]. Furthermore, a cost-effectiveness analysis did not support the addition of bevacizumab [21]. Other inhibitors of VEGF are ramucirumab and aflibercept, but none of these have yet been tested in MPM. A preclinical study on the protein kinase inhibitor nintedanib, which targets three major proangiogenic signalling pathways (VEGF, platelet-derived growth factor and fibroblast growth factor), demonstrated its efficacy against in vitro and in vivo models of MPM [22]. Accordingly, in the randomised phase 2 LUME-Meso trial in chemotherapy naïve patients, the combination of cisplatin-pemetrexed-nintedanib increased PFS compared with the cisplatin-pemetrexed-placebo combination, especially in patients with the epithelioid subtype [23]. These findings are currently being expanded in the phase 3 LUME-Meso trial, whose results are expected this year [24]. However, previous research clearly showed that anti-signalling therapy in combination with cytoreductive chemotherapy should not be given to all patients irrespective of their characteristics, but only to individuals presenting the molecular target of the therapy and in whom these targets are crucial for cancer cell survival. To this end, parallel translation studies should investigate changes in differentially expressed growth factors or proteins in the tumour and tumour microenvironment, as well as in plasma/serum. These studies should provide reliable biomarkers and companion tests for patient selection. 
Where do we go from here? We recommend the following rational clinical development strategy.

1) A phase 1 basket trial of MT95-4 in pretreated patients with different tumour types expressing APN, e.g. mesothelioma, lung, pancreatic and colon cancer, with analysis of activity according to CD13 expression and estimation of a cut-off level for a validated array. One of the baskets could evaluate a combination with cisplatin in an extension cohort.

2) A randomised "pick the winner" phase 2 trial of MT95-4, either as single agent or in combination with cisplatin in treatment-naive patients with a high level of expression of CD13 in their tumour. Selection of tumour type is carried out according to the activity observed in the preceding phase 1 trial.

3) The confirmatory phase 3 trial versus the standard of care backbone chemotherapy, with a hazard ratio for overall survival as the primary end-point according to standardised and validated thresholds [25].

Other possible strategies include a combination of different classes of anti-angiogenic agents in order to observe any incremental or synergistic effect. Possibilities include MT95-4 with either bevacizumab or nintedanib, and MT95-4 with bestatin or tosedostat. These are best explored as parallel randomised phase 2 trials in pretreated patients with high expression of CD13. Previous experience with this class of drugs suggests the compulsory association with a chemotherapeutic agent, for which the addition of MT95-4 may be a double-edged sword, by inhibition of the angiogenic pathway and the survival pathway.

MPM is a notoriously refractory tumour and current recommendations from European and American scientific societies advocate the enrolment of eligible pretreated patients in clinical trials [26-29]. Aminopeptidase antibodies are a new class of agents in this disease. Their adequate development requires their astute use in strategic clinical trials. Exploring their activity and efficacy according to old paradigms may lead to disregarding some of their disruptive characteristics. New wine deserves new sacks.

Conflict of interest: None declared.

\section{References}

1 Otsuki T, Nakashima T, Hamada $\mathrm{H}$, et al. Aminopeptidase N/CD13 as a potential therapeutic target in malignant pleural mesothelioma. Eur Respir J 2018; 51: 1701610.

2 Hitzerd SM, Verbrugge SE, Ossenkoppele G, et al. Positioning of aminopeptidase inhibitors in next generation cancer therapy. Amino Acids 2014; 46: 793-808.

3 Hanahan D, Weinberg RA. Hallmarks of cancer: the next generation. Cell 2011; 44: 646-674.

4 Kumar-Singh S, Vermeulen PB, Weyler J, et al. Evaluation of tumour angiogenesis as a prognostic marker in malignant mesothelioma. J Pathol 1997; 182: 211-216.

5 Sanderink GJ, Artur Y, Siest G. Human aminopeptidases: a review of the literature. J Clin Chem Clin Biochem 1988; 26: 795-807.

6 Wickström M, Larsson R, Nygren P, et al. Aminopeptidase N (CD13) as a target for cancer chemotherapy. Cancer Sci 2011; 102: 501-508.

7 Petrovic N, Schacke W, Gahagan JR, et al. CD13/APN regulates endothelial invasion and filopodia formation. Blood 2007; 110: 142-150.

8 Scornik OA, Botbol V. Bestatin as an experimental tool in mammals. Curr Drug Metab 2001; 2: 67-85.

9 Mina-Osorio P. The moonlighting enzyme CD13: old and new functions to target. Trends Mol Med 2008; 14: 361-371.

10 Löwenberg B, Morgan G, Ossenkoppele GJ, et al. Phase I/II clinical study of Tosedostat, an inhibitor of aminopeptidases, in patients with acute myeloid leukemia and myelodysplasia. J Clin Oncol 2010; 28: 4333-4338.

11 Krige D, Needham LA, Bawden LJ, et al. CHR-2797: an antiproliferative aminopeptidase inhibitor that leads to amino acid deprivation in human leukemic cells. Cancer Res 2008; 68: 6669-6679.

12 Avan A, Narayan R, Giovannetti E, et al. Role of Akt signaling in resistance to DNA-targeted therapy. World J Clin Oncol 2016; 7: 352-369.

13 Giovannetti E, Zucali PA, Assaraf YG, et al. Role of proton-coupled folate transporter in pemetrexed-resistance of mesothelioma:clinical evidence and new pharmacological tools. Ann Oncol 2017; 28: 2725-2732.

14 Giovannetti E, Zucali PA, Assaraf YG, et al. Preclinical emergence of vandetanib as a potent antitumour agent in mesothelioma: molecular mechanisms underlying its synergistic interaction with pemetrexed and carboplatin. $\mathrm{Br} J$ Cancer 2011; 105: 1542-1553.

15 Zalcman G, Mazieres J, Margery J, et al. Bevacizumab for newly diagnosed pleural mesothelioma in the Mesothelioma Avastin Cisplatin Pemetrexed Study (MAPS): a randomised, controlled, open-label, phase 3 trial. Lancet 2016; 387: 1405-1414.

16 Kindler HL, Karrison TG, Gandara DR, et al. Multicenter, double-blind, placebo-controlled, randomized phase II trial of gemcitabine/cisplatin plus bevacizumab or placebo in patients with malignant mesothelioma. J Clin Oncol 2012; 30: 2509-2515.

17 Sandler A, Gray R, Perry MC, et al. Paclitaxel-carboplatin alone or with bevacizumab for non-small-cell lung cancer. N Engl J Med 2006; 355: 2542-2550.

18 Reck M, von Pawel J, Zatloukal P, et al. Overall survival with cisplatin-gemcitabine and bevacizumab or placebo as first-line therapy for nonsquamous non-small-cell lung cancer: results from a randomised phase III trial (AVAiL). Ann Oncol 2010; 21: 1804-1809.

19 Ceresoli GL, Zucali PA, Mencoboni M, et al. Phase II study of pemetrexed and carboplatin plus bevacizumab as first-line therapy in malignant pleural mesothelioma. Br J Cancer 2013; 109: 552-558. 
20 National Comprehensive Cancer Network. NCCN guidelines. www.nccn.org/professionals/physician_gls/pdf/mpm. pdf Date last accessed: April 2, 2018.

21 Zhan $\mathrm{M}$, Zheng $\mathrm{H}, \mathrm{Xu} \mathrm{T}$, et al. Cost-effectiveness analysis of additional bevacizumab to pemetrexed plus cisplatin for malignant pleural mesothelioma based on the MAPS trial. Lung Cancer 2017; 110: 1-6.

22 Laszlo V, Ozsar J, Klikovits T, et al. Preclinical investigation of the therapeutic potential of nintedanib in malignant pleural mesothelioma. Proceedings of the 13th International Conference of the International Mesothelioma Interest Group 2016; Abstract PP02.61.

23 Grosso F, Steele N, Novello S, et al. Nintedanib plus pemetrexed/cisplatin in patients with malignant pleural mesothelioma: phase II results from the randomized, placebo-controlled LUME-Meso trial. J Clin Oncol 2017; 35: 3591-3600.

24 Boehringer Ingelheim. LUME-Meso: double blind, randomised, multicentre, phase II/III study of nintedanib in combination with pemetrexed/cisplatin followed by continuing nintedanib monotherapy versus placebo in combination with pemetrexed/cisplatin followed by continuing placebo monotherapy for the treatment of patients with unresectable malignant pleural mesothelioma. https://clinicaltrials.gov/ct2/show/NCT01907100 Date last updated: March 13, 2018. Date last accessed: April 28, 2018.

25 Cherny NI, Sullivan R, Dafni U, et al. A standardised, generic, validated approach to stratify the magnitude of clinical benefit that can be anticipated from anti-cancer therapies: the European Society for Medical Oncology Magnitude of Clinical Benefit Scale (ESMO-MCBS). Ann Oncol 2015; 26: 1547-1573.

26 Scherpereel A, Astoul P, Baas P, et al. Guidelines of the European Respiratory Society and the European Society of Thoracic Surgeons for the management of malignant pleural mesothelioma. Eur Respir J 2010; 35: 479-495.

27 Baas P, Fennell D, Kerr KM, et al. Malignant pleural mesothelioma: ESMO clinical practice guidelines for diagnosis, treatment and follow-up. Ann Oncol 2015; 26: Suppl. 5, v31-v39.

28 Woolhouse I, Bishop L, Darlison L, et al. British Thoracic Society Guideline for the investigation and management of malignant pleural mesothelioma. Thorax 2018; 73: Suppl. 1, i1-i30.

29 Kindler HL, Ismaila N, Armato SG, et al. Treatment of malignant pleural mesothelioma: American Society of Clinical Oncology Clinical Practice Guideline. J Clin Oncol 2018; 36: 1343-1373. 